\title{
Local Quality Method for the Iris Image Pattern
}

\author{
Luis Miguel Zamudio-Fuentes ${ }^{1}$, Mireya S. García-Vázquez ${ }^{1}$, \\ and Alejandro Alvaro Ramírez-Acosta ${ }^{2}$ \\ ${ }^{1}$ Centro de Investigación y Desarrollo de Tecnología Digital (CITEDI-IPN), \\ Avenida del Parque 1310, Tijuana, B.C. México 22510 \\ ${ }^{2}$ MIRAL. R\&D, 1047 Palm Garden, Imperial Beach, 91932 USA \\ \{zamudio,mgarciav\}@citedi.mx, ramacos10@hotmail.com
}

\begin{abstract}
Recent researches on iris recognition without user cooperation have introduced video-based iris capturing approach. Indeed, it provides more information and more flexibility in the image acquisition stage for noncooperative iris recognition systems. However, a video sequence can contain images with different level of quality. Therefore, it is necessary to select the highest quality images from each video to improve iris recognition performance. In this paper, we propose as part of a video quality assessment module, a new local quality iris image method based on spectral energy analysis. This approach does not require the iris region segmentation to determine the quality of the image such as most of existing approaches. In contrast to other methods, the proposed algorithm uses a significant portion of the iris region to measure the quality in that area. This method evaluates the energy of 1000 images which were extracted from 200 iris videos from the MBGC NIR video database. The results show that the proposed method is very effective to assess the quality of the iris information. It obtains the highest 2 images energies as the best 2 images from each video in 226 milliseconds.
\end{abstract}

Keywords: Iris recognition, biometrics, video, quality assessment.

\section{Introduction}

According to the literature, considerable improvement in recognition performance is possible when poor quality images are removed using image quality assessment stage in an automated biometric system [1]. Motivated by this idea, we propose as part of a video quality assessment module that our research group is currently developing [2-4, 7], a new local quality iris image method based on spectral energy analysis. The figure 1 shows how the iris video sequence is first globally analyzed to quickly determine the quality of the whole image and obtain a small quality image set [4]. Secondly, the images resulting from that analysis are used for local quality assessment to obtain the best two images from a video that are optimal to continue with the recognition module.

Existing approaches about local iris quality measures consist of extracting the iris region from the image based on segmentation algorithm, and then evaluate the quality of this area using different bands of the Mexican hat wavelet [5]. Similarly, after 
segmentation stage, other authors localize two small regions near to the limit of the pupil to measure the quality applying the third-level wavelet transformation of the two selected regions [6]. Those researches considered that the region near the edge of the pupil was the area that contained more feature information of the iris.

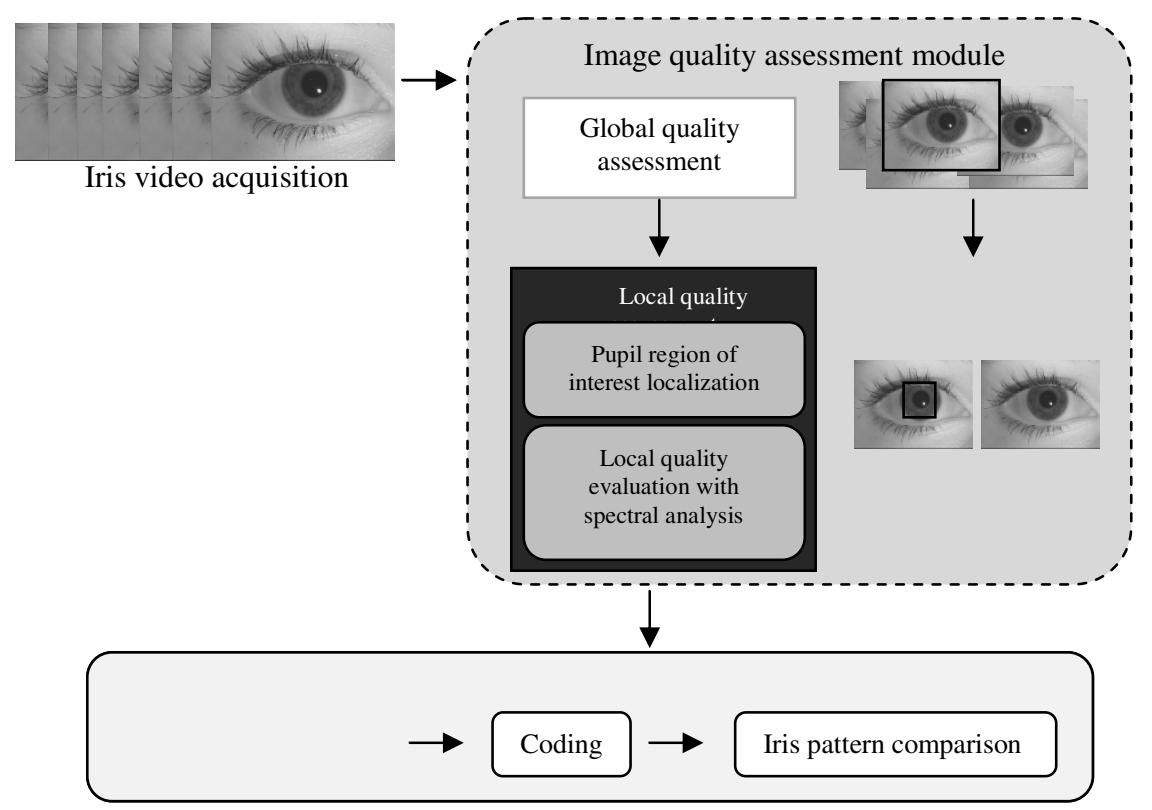

Fig. 1. Typical iris recognition system with video-based iris capturing that includes the proposed image quality assessment module

In this paper, our proposition also examines the local quality in the region near the pupil, but without iris segmentation stage. Note that in this work, the pupil region of interest (PROI) is automatically located. A square region of interest (SROI) which covers the majority features of iris information is determined from PROI central point. Then SROI is used to analyze the high and low frequencies from all pixel intensities values.

In summary, the local quality assessment stage evaluates all images coming from the global stage (previous work realized in $[2,4,7]$ ). In this phase, the area of PROI is first dynamically determined. Secondly, the SROI is obtained from PROI central point, and then this information is used to analyze the quality of the SROI using Kang's Kernel [1, 8, and 9]. It is important to highlight that in our previous work [7], Kang's kernel was superior to other kernels in showing less computing time and more efficiency performance.

The remainder of this paper is organized as follows. Section 2 reviews the local quality assessment. Continue in section 3 with the implementation and results. Finally, in section 4, we draw the conclusions. 


\section{Local Quality Stage}

The main features of the iris information are located near to the boundary of the pupil. Therefore, it is important in all iris recognition systems to ensure the biometric information quality in that region. According to [7], the iris image quality is determinate by two factors: defocus and blur. These factors are considered visually similar. However, they differ in how they are generated. Defocus is an optic aberration in which an image is simply out of focus. On the other hand, blur is produced by the relative displacement between the object and the camera. These factors can be analyzed and detected through a kernel that measures the image energy. The energy of a defocused or blurred image concentrates on the lower frequency part. Hence, spectral analysis of the frequency suggests that an effective way to estimate the degree of focus is measuring its total energy at higher spatial frequencies. The quality assessment in our system begins with a global analysis of all images in an iris video, in which the whole image is evaluated with a kernel to rapidly select a set of high quality images as it is described by Colores et al in [7]. It is important to note that the global stage provides a rough good quality images. Nevertheless, in some regions of the iris texture can remain important imperfections such as blur, defocus, reflection, etc. To ensure that the iris region is free of imperfections, this paper proposes to add the stage of local quality to the image quality assessment module. In this stage, all images coming from the global stage are processed.

\section{2a Pupil Region of Interest Localization}

During the video acquisition, the position of the pupil may differ from one image to other. For that reason, it is important to dynamically localize the PROI in the image. This region is used to make different processes in iris images like: to detect and eliminate the pupil, to localize and extract the iris region and particularly local quality assessment. As it is known, the intensity of the pupil is close to zero in a grayscale [10]. Using this principle, we developed a basic histogram analysis method of grayscale levels to find the maximum and minimum intensity values. This simple method shows information about how the intensities are distributed around the image.

To dynamically determine the pupil region of interest we propose the following algorithm, which localizes the minimum and maximum values over the histogram. First, a histogram is obtained using the intensities of each image resulting from the global quality analysis (Figure 2a). Secondly, an average filter is applied to smooth the curve generated; this is in order to eliminate false peaks and valleys (Figure 2b). Thirdly the slopes of the curve are calculated to determine any positive to negative change and vice versa. The observed changes are used to locate and determine the position of the lobes or peaks and valleys of the histograms (Figure 2c). 


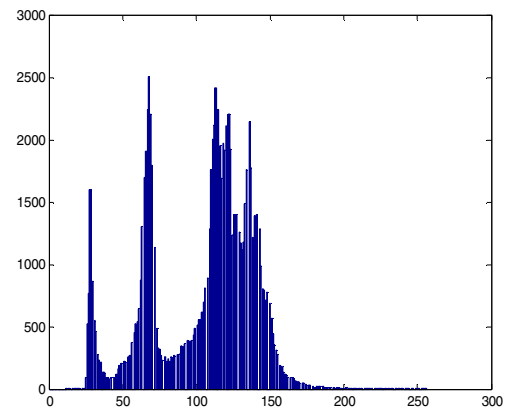

a)

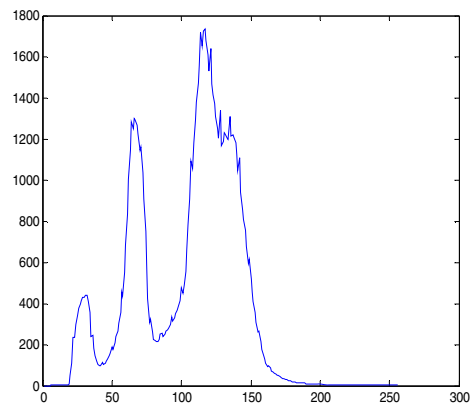

b)

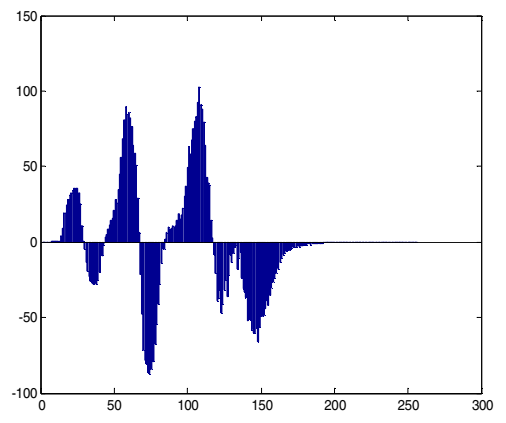

c)

Fig. 2. Histogram analysis

The position of the first peak value is considered as the intensity value of the pupil. To dynamically determine the PROI, the following inequality is evaluated to localize the positions of the pixels belonging to the pupil,

$$
f(x, y)=\left\{\begin{array}{cc}
255, & I(x, y)<h_{1} \\
I(x, y), & \text { otherwise }
\end{array}\right.
$$

where $f(x, y)$ is the resulting image, $I(x, y)$ is the intensity value of each pixel of the image and $h_{1}$ is the value of the first peak encountered in the implementation of the algorithm. The next step is to extract all $x, y$ positions from the pixels that changed its intensity to 255 . To calculate the pseudo-center of the PROI, we obtain the average positions of $x$ and $y$ using equation 2 ,

$$
\text { center }_{x}=\frac{1}{k} \sum_{i=1}^{k} \operatorname{pos}_{x}(i), \text { center }_{y}=\frac{1}{k} \sum_{i=1}^{k} \operatorname{pos}_{y}(i)
$$

where $\operatorname{pos}_{x}(i)$ and $\operatorname{pos}_{y}(i)$ are the $x, y$ positions and $k$ is the total number of pixels that changed the intensity value. Once the pseudo-center is calculated, the algorithm determines a square area of size 160x160 pixels called square region of interest 
(SROI). Figure 3 shows, two examples of this region. After localizing the dynamic search area, the next step is the assessment of the local quality with spectral analysis, which is described in the next section.

The pupil area is not considered into the local quality assessment.

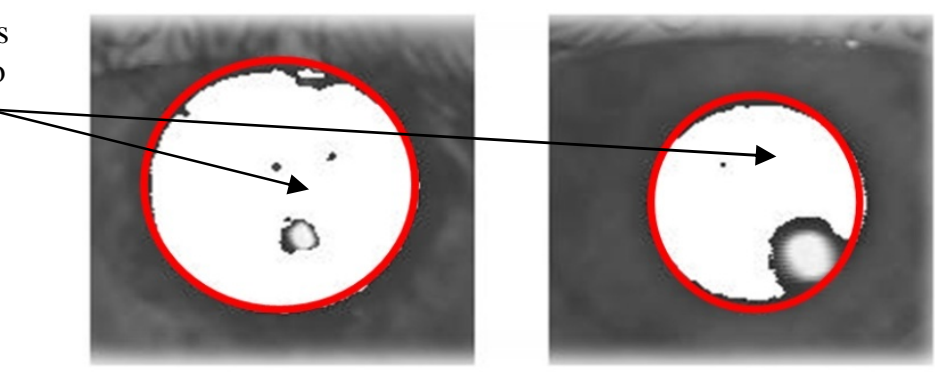

Fig. 3. Square region of interest (SROI)

\section{2b Local Quality Evaluation with Spectral Analysis}

Once the SROI is obtained, this area is convolved with a kernel to evaluate the focus degree existing in that region. There are many kernels to perform this evaluation, the kernel of Daugman [11], Byung et al, and Kang et al [1, 8, 9]. However, to carry out the local quality assessment a Kang's kernel is conducted (Figure 4). This election was done after analyzing the results presented by Colores et al [7]. In this work, it was shown that Kang's kernel takes less time that the Daugman's kernel.

\begin{tabular}{ccccc}
\hline-1 & -1 & -1 & -1 & -1 \\
\hline-1 & -1 & 4 & -1 & -1 \\
-1 & 4 & 4 & 4 & 3 \\
-1 & -1 & 4 & -1 & 3 \\
-1 & -1 & -1 & -1 & -1 \\
\hline
\end{tabular}

a)

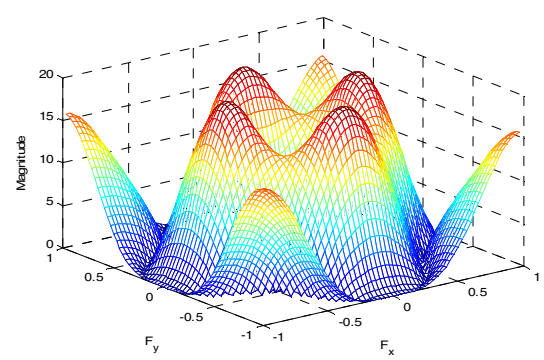

b)

Fig. 4. a) 5x5 Kang's kernel. b) The frequency response of the kernel (Fourier spectrum).

To perform the local quality assessment, this kernel is convolved with the SROI according to the following equation in the space domain:

$$
\operatorname{imfilter}(x, y)=\operatorname{sroi}(x, y) * k(x, y)=\sum_{m=0}^{M-1} \sum_{n=0}^{N-1} \operatorname{sroi}(m, n) k(x-m, y-n)
$$


where imfilter $(x, y)$ is the filtered image, $\operatorname{sroi}(x, y)$ is the square region of interest, $k(x, y)$ is Kang's kernel. According to the convolution theorem [12], the convolution product reduces in the frequency domain to a simple product, as is shown in equation 4 :

$$
\operatorname{IMFILTER}(u, v)=\operatorname{SROI}(u, v) \cdot K(u, v)
$$

The filtered image $\operatorname{IMFILTER}(u, v)$ is obtained by multiplying the square region of interest $\operatorname{SROI}(u, v)$ and Kang's kernel $K(u, v)$ [1, 8-9] to estimate the degree of focus. As a result of using the mentioned theorem, the computation time is reduced in the frequency domain. After multiplication, the inverse Fourier transform is calculated and the energy of the image through Parseval's theorem using the equations 5 and 6 to obtain the spectral energy density [13] (energy). Finally, a threshold is applied to determine the quality of the square region of interest (eq.7),

$$
\begin{gathered}
\operatorname{imfilter}(x, y)=F^{-1}\{\operatorname{IMFILTER}(u, v)\} \\
\text { ene }=|\operatorname{imfilter}|^{2} \\
\text { ene }>T
\end{gathered}
$$

ene is the energy of the $\operatorname{SROI}(u, v)$ and $T$ is the threshold applied to determine the quality of the SROI.

\section{Implementation and Results}

The local quality assessment method is tested with 1000 images of 200 videos from the MBGC NIR video database [14]. The data set selected were acquired using an Iridian LG EOU 2200 camera. Each video consist of 300 to 400 eye frames with a resolution of 480 by 640 pixels in 8 bits-grayscale space (intensity values ranging from 0 to 255) at 30 frames per second (fps). For each video, 5 images are extracted using the global quality assessment image set of our previous work [2, 4, 7] (see fig. 1). These 5 images are randomly chosen by making a subset from the image quality set, and then one of these is selected for each subset.

The following describes the algorithm developed for the local quality assessment:

- Locate the pupil region of interest for the five images from each video.

- Determine the square region of interest SROI.

- Take the intensities values of the SROI and obtain the corresponding values in the frequency domain as the result of the DFT (Discrete Fourier Transform).

- Calculate the Kang's kernel value in the frequency domain using the DFT.

- Perform the convolution operation between the SROI and $K$, using the convolution theorem of Fourier transforms (Eq. 4).

- Use Equation 5 and calculate the inverse Discrete Fourier Transform to obtain its value in the space domain of the result IMFILTER.

- Calculate the energy of the result using the equation 6. 
- Evaluate the inequality of equation 7 and select the highest two energies from the five energies, which are highest quality images of the video. Then continue with the pre-processing stage of the recognition system [2].

After computing the algorithm, the results are concentrated in Table 1. It shows the energy obtained in the local quality assessment for the first 30 videos of the 200 videos from the MBGC used in this evaluation. The table has twelve columns, from left to right the first column represents the video number and the next five columns are the energies by image from the first fifteen videos. The next six rows represent the rest energies of the other videos. It is observed that the majority of the energies are around 90 and $120 \mathrm{~J} / \mathrm{Hz}$ that means the photon flux [15]. However, this method obtains the highest two energies which are highlighted in Table 1 to identify the best images from a video sequence. On the other hand, most of the highest energies are among images 3, 4 and 5. All results are available upon request.

Table 1. Spectral energy density for each video

\begin{tabular}{|c|c|c|c|c|c|c|c|c|c|c|c|}
\hline & \multicolumn{5}{|c|}{ Energy } & \multicolumn{5}{|c|}{ Energy } \\
\hline Video & im 1 & im2 & im 3 & im 4 & im 5 & Video & im 1 & im 2 & im3 & im 4 & im5 \\
\hline 1 & 103.17 & 109.61 & 110.23 & $\mathbf{1 1 3 . 0 7}$ & $\mathbf{1 1 3 . 8 5}$ & 16 & 116.31 & 115.09 & $\mathbf{1 2 0 . 1 1}$ & $\mathbf{1 1 6 . 3 4}$ & 111.45 \\
\hline 2 & 80.16 & 89.18 & $\mathbf{9 8 . 4 1}$ & 93.79 & $\mathbf{1 0 3 . 3 8}$ & 17 & $\mathbf{9 9 . 3 0}$ & $\mathbf{9 2 . 9 9}$ & 92.44 & 92.58 & 88.60 \\
\hline 3 & 101.16 & 109.79 & $\mathbf{1 1 1 . 3 2}$ & $\mathbf{1 1 3 . 7 0}$ & 109.05 & 18 & 105.63 & 100.80 & $\mathbf{1 0 1 . 0 5}$ & 98.16 & $\mathbf{1 0 1 . 7 9}$ \\
\hline 4 & 77.20 & $\mathbf{9 5 . 1 3}$ & 93.88 & 91.42 & $\mathbf{9 4 . 2 7}$ & 19 & 96.06 & 91.95 & $\mathbf{1 1 0 . 9 4}$ & $\mathbf{1 1 2 . 1 6}$ & 91.04 \\
\hline 5 & 95.47 & 97.50 & $\mathbf{1 0 7 . 1 4}$ & 106.15 & $\mathbf{1 1 0 . 4 4}$ & 20 & $\mathbf{1 1 1 . 5 3}$ & $\mathbf{1 1 4 . 7 8}$ & 89.74 & 107.95 & 104.94 \\
\hline 6 & 87.18 & 96.46 & $\mathbf{9 8 . 3 6}$ & 96.65 & $\mathbf{1 0 0 . 4 8}$ & 21 & $\mathbf{1 0 9 . 9 5}$ & $\mathbf{1 1 3 . 0 7}$ & 111.99 & 108.71 & 105.60 \\
\hline 7 & 99.77 & 96.00 & $\mathbf{1 0 8 . 0 7}$ & $\mathbf{1 0 1 . 6 8}$ & 100.93 & 22 & 97.23 & 104.02 & 103.39 & $\mathbf{1 1 1 . 5 6}$ & $\mathbf{1 0 7 . 5 8}$ \\
\hline 8 & 91.92 & 91.78 & $\mathbf{1 0 5 . 8 2}$ & 90.13 & $\mathbf{8 8 . 0 3}$ & 23 & $\mathbf{1 4 9 . 8 0}$ & 136.59 & 140.43 & $\mathbf{1 4 9 . 8 4}$ & 124.70 \\
\hline 9 & 103.96 & 107.79 & 111.56 & $\mathbf{1 1 4 . 4 2}$ & $\mathbf{1 1 3 . 2 0}$ & 24 & 124.37 & 142.21 & $\mathbf{1 5 6 . 3 5}$ & $\mathbf{1 5 2 . 3 8}$ & 103.39 \\
\hline 10 & 98.86 & $\mathbf{1 1 3 . 1 6}$ & 104.10 & $\mathbf{1 1 1 . 1 5}$ & 109.69 & 25 & 98.84 & 101.68 & $\mathbf{1 0 4 . 2 7}$ & $\mathbf{1 0 3 . 7 8}$ & 91.15 \\
\hline 11 & 92.15 & 99.36 & $\mathbf{1 0 0 . 2 4}$ & 96.26 & $\mathbf{1 0 3 . 5 7}$ & 26 & 106.33 & $\mathbf{1 1 3 . 8 4}$ & $\mathbf{1 1 0 . 7 9}$ & 109.67 & 106.16 \\
\hline 12 & 102.72 & 96.99 & $\mathbf{1 0 6 . 6 2}$ & $\mathbf{1 0 8 . 2 9}$ & 102.81 & 27 & $\mathbf{1 2 0 . 1 8}$ & $\mathbf{1 2 4 . 0 8}$ & 114.04 & 111.64 & 118.90 \\
\hline 13 & $\mathbf{1 0 8 . 7 8}$ & 100.70 & $\mathbf{1 1 1 . 8 0}$ & 99.36 & 98.37 & 28 & 109.61 & $\mathbf{1 1 4 . 7 1}$ & 97.37 & 102.58 & $\mathbf{1 1 5 . 3 7}$ \\
\hline 14 & 111.37 & $\mathbf{1 1 2 . 6 5}$ & $\mathbf{1 1 1 . 6 1}$ & 109.39 & 108.15 & 29 & 90.20 & 101.27 & $\mathbf{1 0 8 . 7 4}$ & 104.03 & $\mathbf{1 1 0 . 9 8}$ \\
\hline 15 & 91.45 & $\mathbf{1 0 0 . 7 3}$ & 91.98 & 88.49 & $\mathbf{9 3 . 4 5}$ & 30 & $\mathbf{1 4 3 . 7 3}$ & 110.40 & $\mathbf{1 2 6 . 6 2}$ & 114.30 & 106.33 \\
\hline
\end{tabular}

To illustrate an example of this assessment, the video number 23 or $05303 \mathrm{~d} 273$ as is label in the MBGC is used in Figure 5. Image 1 and image 4 show a sharp and distinguishable iris pattern. In contrast, in the images 2 and 3 the iris is not distinguishable and image 5 is not sharp enough.

In Table 2 important differences between the proposed and the existing approaches $[5,6]$ are pointed out. For instance, the iris information used in the existing approaches came from CASIA [16] iris database, which is acquired under controlled environment. The subject is steady at the acquisition time and the quality of the images is optimal for recognition. While in the proposed method, the iris information is extracted from the MBGC NIR video database, which is provided as a challenge, since it was acquired under unconstraint environment and the subjects were walking at the acquisition time. The proposed method uses a significant number of iris pixels to assess the local quality, in average $200 \%$ more iris texture information than Yi Chen, Yi Huang et al [5, 6], and in contrast to them, it doesn't need iris segmentation. 


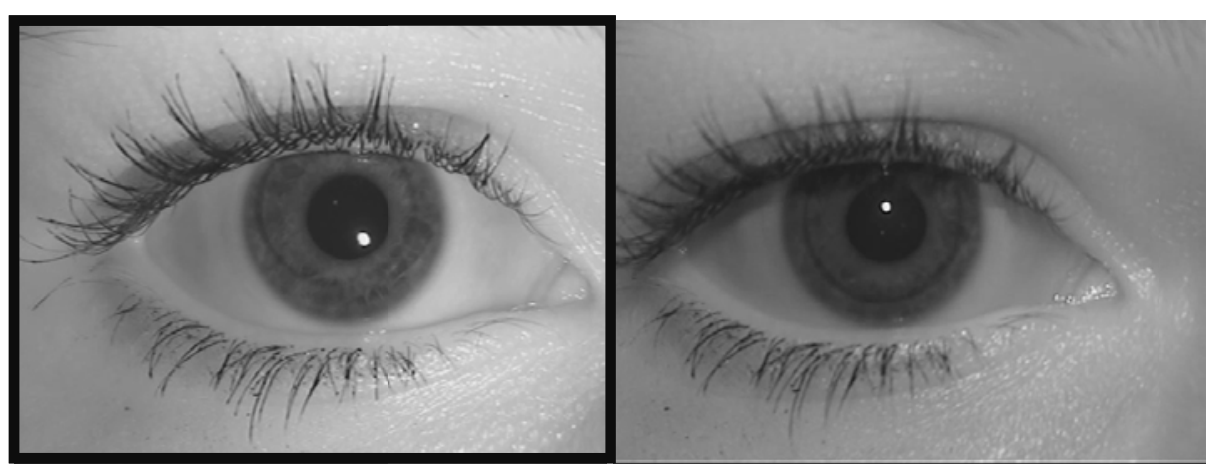
im1
im2

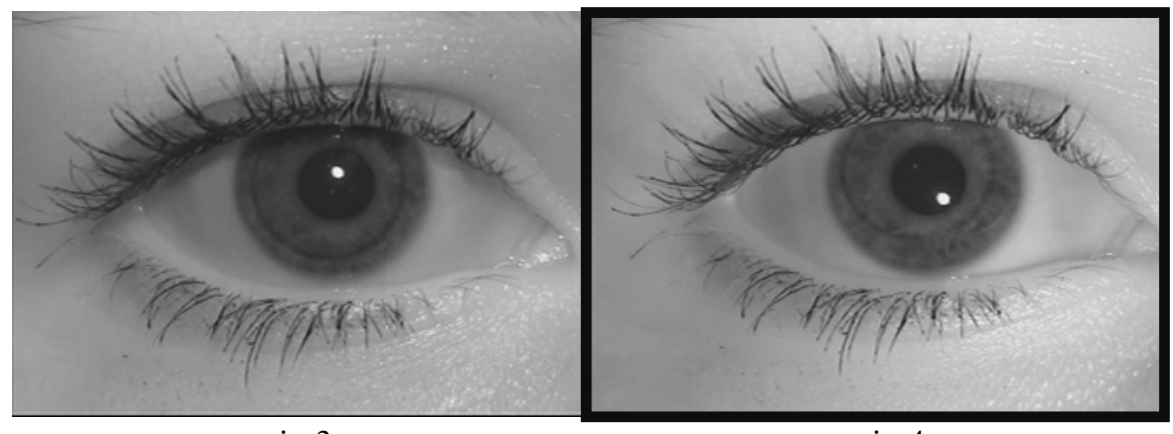

$\operatorname{im} 3$ im4

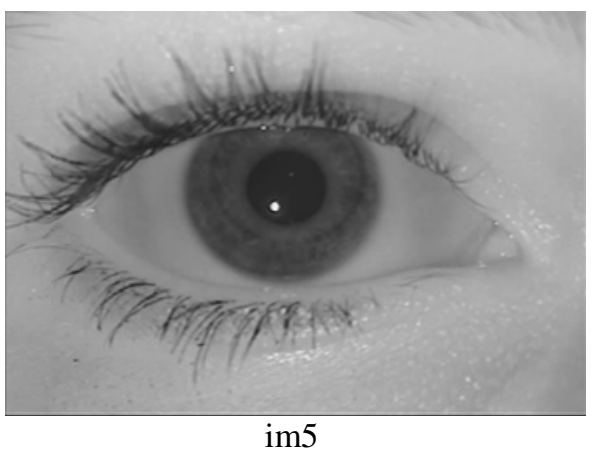

Fig. 5. Example of the local quality assessment

In addition, according to [7], the Park's kernel (which is used in our proposed module to assess the quality of the iris information), is more effective in computing time and produce less FAR and FRR than Continuous Wavelet Transform. Even though our propose method is a fast quality assessment module that just takes $226 \mathrm{~ms}$, we don't discard the stages added in [6] which could be optimized and included for future work to improve the module. Finally, the accuracy of the evaluated methods depends on SROI selection in the case of the proposed method, iris segmentation in [5], and the painted eyelid and eyelashes for the case [6]. 
Table 2. Comparison between the proposed method and existing approaches

\begin{tabular}{|l|l|l|l|}
\hline & Proposed & Yi Chen et al [5] & Yi Huang et al [6] \\
\hline Iris information from & Video & Images & Image sequence \\
\hline Database & MBGC[14] & CASIA[16] & CASIA[16] \\
\hline Resolution & $640 x 480$ & $320 x 280$ & $320 x 280$ \\
\hline Energy evaluation & Park's Kernel & Mexican Hat & Continuous Wavelets transform \\
\hline Number of iris pixels evaluated & $\sim 21252$ & Not specified & 6144 \\
\hline Iris segmentation & No & Yes & Yes \\
\hline \# video & 200 & No & 300 \\
\hline \# images & 1000 & 2608 & 3600 \\
\hline Quality assess time & $226 \mathrm{~ms}$ & Not specified & Not specified \\
\hline Inaccuracy when & $\begin{array}{l}\text { Bad SROI } \\
\text { selection }\end{array}$ & $\begin{array}{l}\text { Bad iris } \\
\text { segmentation }\end{array}$ & $\begin{array}{l}\text { Spectacled iris images, } \\
\text { Iris affected by pained } \\
\text { eyelashes or eyelid }\end{array}$ \\
\hline Platform & $\begin{array}{l}\text { Matlab } \\
\text { R2008b }\end{array}$ & Not specified & Matlab 7.0̈̈ \\
\hline
\end{tabular}

The proposed method was tested using Matlab R2008b in a $2.2 \mathrm{Ghz}$ Pentium dual core computer with $3 \mathrm{~Gb}$ of ram. In addition, it is not optimized to work in real time. However, the computing time that will take to assess the local quality for video sequence is in average 226 milliseconds. Therefore, it could be optimized for real time automatic iris recognition systems.

\section{Conclusions}

In this paper, we propose and evaluate an algorithm for local quality assessment as part of a video quality assessment module. In contrast to other methods, it uses approximately $200 \%$ more significant portion of the iris region to determine the quality in that area. This method does not need the segmentation of the iris region to determine the quality of the image. In addition, the proposed method is suitable for real time optimization in automatic iris recognition systems. It takes just only $226 \mathrm{~ms}$ to access the local quality for each video sequence. In the MBGC video database, according Table 1, most of the highest energies are among images 3, 4 and 5. It means that in the acquisition step, the first images that were acquired are not in the focus plane. The results showed that the proposed algorithm is fast and very effective to identify whether the iris region is out of focus or not. Furthermore, it obtains two images of the highest quality from a video sequence. These two images provide more reliable information which is useful to further stage of the recognition system. With the conjunction of the video quality assessment module through the global and the local quality assessment we are ensuring that the iris region is focused. Consequently, the accuracy and the robustness of the system will increase by minimizing the false reject rate (FRR) and false acceptation rate (FAR). On the other hand, the accuracy of this method depends on the automatically selection of the SROI. For future improvement, we are going to analyze other quality factor as pupil dilatation, segmentation accuracy, iris information ratio and obtain the relation between FRR and FAR. 
Acknowledgment. This research was supported by grant SIP20110032 from IPNCITEDI.

\section{References}

[1] Kang, B.J., Park, K.R.: A study on fast iris restoration based on focus checking. In: Perales, F.J., Fisher, R.B. (eds.) AMDO 2006. LNCS, vol. 4069, pp. 19-28. Springer, Heidelberg (2006)

[2] Zamudio-Fuentes, L.M., García-Vázquez, M.S., Ramírez-Acosta, A.A.: Iris Segmentation Using a Statistical Approach. In: Martínez-Trinidad, J.F., Carrasco-Ochoa, J.A., Kittler, J. (eds.) MCPR 2010. LNCS, vol. 6256, pp. 164-170. Springer, Heidelberg (2010)

[3] García-Vázquez, M., Ramírez-Acosta, A.: Person verification process using iris information. Research in Computing Science 44, 97-104 (2009)

[4] Zamudio, L.M.: Reconocimiento del iris como identificación biométrica utilizando el video. MSc thesis, IPN (January 2011)

[5] Chen, Y., Dass, S.C., Jain, A.K.: Localized iris image quality using 2-d wavelets. In: ICB 2006 (2006)

[6] Huang, Y., Ma, Z., Xie, M.: Rapid and effective method of quality assessment on sequence iris image. In: MIPPR 2007. Proc. of SPIE, vol. 6786 (2007)

[7] Colores-Vargas, J.M., García-Vázquez, M.S., Ramírez-Acosta, A.A.: Measurement of defocus level in iris images using different convolution kernel methods. In: MartínezTrinidad, J.F., Carrasco-Ochoa, J.A., Kittler, J. (eds.) MCPR 2010. LNCS, vol. 6256, pp. 125-133. Springer, Heidelberg (2010)

[8] Kang, B.J., Park, K.R.: Real-time image restoration for iris recognition Systems. IEEE Trans. on Systems 37(6), 1555-1566 (2007)

[9] Kang, B.J., Park, K.R.: A study on restoration of iris images with motion-and-optical blur on mobile iris recognition devices. Wiley Periodicals (2009)

[10] Lee, Y., Phillips, P.J., Micheals, R.J.: An Automated Video-Based System for Iris Recognition. In: Tistarelli, M., Nixon, M.S. (eds.) ICB 2009. LNCS, vol. 5558, pp. 1160 1169. Springer, Heidelberg (2009)

[11] Daugman, J.G.: How iris recognition works. IEEE Trans. Circ. Syst. Video Tech. 14(1), 21-30 (2004)

[12] Gonzalez, R.C., Woods, R.: Digital image processing. Addison-Wesley (1996)

[13] Correlation and Spectral Density, http://www.ensc.sfu.ca/ jiel/courses/327/bin/pdf /

Pre_15_Rxx.pdf

[14] Multi Biometric Grand Challenge, http://face.nist.gov/mbgc

[15] Image formation,

http://www.cs.toronto. edu/ fleet/courses/2503/fall10/

Handouts / imageFormation.pdf

[16] Chinese Academy of Sciences, Institute of Automation (CASIA), http://figment.cse.usf.edu/ sfefilat/data/papers/ WeBCT9.29.pdf 